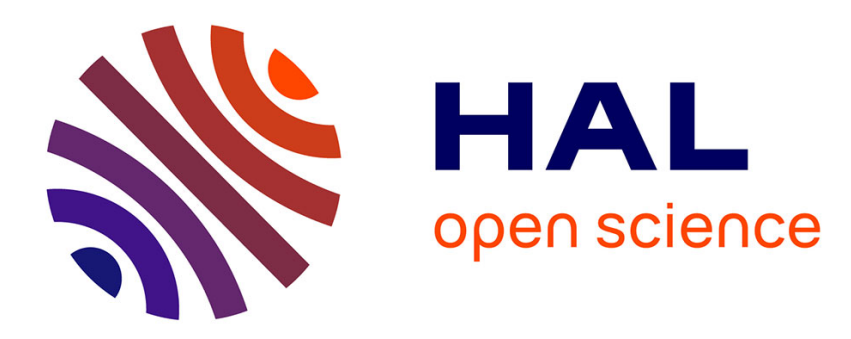

\title{
Propriétés magnétiques de l'alliage amorphe Gd54Ag46
}

B. Boucher

\section{To cite this version:}

B. Boucher. Propriétés magnétiques de l'alliage amorphe Gd54Ag46. Journal de Physique Lettres, 1976, 37 (12), pp.345-347. 10.1051/jphyslet:019760037012034500 . jpa-00231308

\section{HAL Id: jpa-00231308 https://hal.science/jpa-00231308}

Submitted on 1 Jan 1976

HAL is a multi-disciplinary open access archive for the deposit and dissemination of scientific research documents, whether they are published or not. The documents may come from teaching and research institutions in France or abroad, or from public or private research centers.
L'archive ouverte pluridisciplinaire HAL, est destinée au dépôt et à la diffusion de documents scientifiques de niveau recherche, publiés ou non, émanant des établissements d'enseignement et de recherche français ou étrangers, des laboratoires publics ou privés. 


\title{
PROPRIÉTÉS MAGNÉTIQUES DE L'ALLIAGE AMORPHE $\mathbf{G d}_{54} \mathbf{A g}_{46}$
}

\author{
B. BOUCHER \\ Service de Physique du Solide et de Résonance Magnétique \\ C. E. N. Saclay, B.P. no 2, 91190 Gif-sur-Yvette, France \\ (Reçu le 11 août 1976, révisé le 9 septembre 1976, accepté le 15 septembre 1976)

\begin{abstract}
Résumé. - On a mesuré la susceptibilité magnétique et la variation de l'aimantation en fonction d'un champ appliqué à différentes températures comprises entre 6 et $300 \mathrm{~K}$. On montre que l'alliage amorphe $\mathrm{Gd}_{54} \mathrm{Ag}_{46}$ présente une aimantation spontanée au-dessous de $122 \mathrm{~K}$, que les interactions magnétiquies sont en grande majorité ferromagnétiques, mais que certaines sont, par suite du désordre topologique, antiferromagnétiques. Il est probable que l'ordre magnétique, qui s'établit à basse température, puisse exister sans formation de domaines magnétiques.
\end{abstract}

\begin{abstract}
The magnetic susceptibility and the variation of magnetization with applied field at different temperatures (between 6 and $300 \mathrm{~K}$ ) have been measured. The amorphous $\mathrm{Gd}_{54} \mathrm{Ag}_{46}$ alloy shows a spontaneous magnetization below $122 \mathrm{~K}$. The magnetic interaction is for the most part ferromagnetic, but there exists also an antiferromagnetic interaction because of topological disorder. It is possible that the magnetic order observed at low temperature exists without the formation of magnetic domains.
\end{abstract}

Dans un récent article [1], nous avons décrit les propriétés magnétiques de l'alliage amorphe $\mathrm{Tb}_{52} \mathrm{Ag}_{48}$. Au-dessous de $64 \mathrm{~K}$ cet alliage présente une aimantation spontanée et au-dessous de $40 \mathrm{~K}$, l'anisotropie locale due au champ électrique créé par les ions voisins est du même ordre de grandeur que l'échange ferromagnétique. A 4,4 $\mathrm{K}$ il n'est pas possible d'atteindre la saturation, même avec des champs de $400 \mathrm{kOe}$. Des propriétés analogues ont été observées sur des alliages ferrimagnétiques tels que $\mathrm{TbFe}_{2}[2,3]$. Pour expliquer les propriétés, Harris et coll. [4] ont développé un modèle faisant intervenir une anisotropie locale forte. Ils ont montré que cette anisotropie est très probablement uniaxe et que les directions d'anisotropie sont distribuées de façon aléatoire. Le cas de TbAg présente l'avantage de ne comporter qu'un seule type d'ion magnétique $\mathrm{Tb}^{3+}$. Les moments sont soumis à des interactions d'échange ferromagnétiques qui tendent à les aligner dans le même sens et à une anisotropie locale (probablement uniaxe). Les directions d'anisotropie, corrélées ou non à courte distance, sont réparties dans tout l'angle solide. Cette anisotropie tend à orienter les moments de ion $\mathrm{Tb}^{3+}$ suivant toutes les directions de l'angle solide. La compétition entre l'échange et l'anisotropie entraîne l'existence, à basse température, d'un ordre magnétique que nous appelons ferromagnétique, en ce sens que les mesures mettent en évidence une aimantation spontanée de plusieurs magnétons de Bohr par atome, c'est-à-dire que les moments tendent à s'aligner dans le même sens sous l'influence de l'échange, mais que cet alignement n'est pas parfait, les moments gardant une certaine dispersion les uns par rapport aux autres sous l'influence de l'anisotropie.

Afin de préciser le rôle joué par l'échange et celui joué par l'anisotropie dans l'établissement de l'ordre magnétique, nous avons étudié l'alliage amorphe GdAg. L'ion $\mathrm{Gd}^{3+}$ étant un ion $\mathrm{S}$, il ne doit pas présenter, sous l'influence du champ électrostatique créé par ses voisins, d'anisotropie; seul l'échange doit intervenir dans l'ordre magnétique.

1. Préparation. - L'échantillon a été préparé par pulvérisation cathodique suivant la méthode exposée précédemment $[1,5]$ à partir de l'alliage cristallisé $\mathrm{Gd}_{50} \mathrm{Ag}_{50}$ ( $\mathrm{Gd}$ de pureté $99,9 \%$ et $\mathrm{Ag}$ de pureté $99,99 \%$ ). Le produit obtenu sous forme de lame épaisse (80 à $100 \mu)$ a été analysé avec une microsonde. La composition de l'alliage èst $\mathrm{Gd}_{54} \mathrm{Ag}_{46}$. C'est avec cette formule que nous avons interprété toutes les mesures faites sur le produit finement broyé. Les échantillons ont été examinés par diffraction de RX et d'électrons. Aucune cristallite (>20 $\AA$ ) n'a pu être mise en évidence.

2. Mesures magnétiques. - Nous avons mesuré la susceptibilité avec une balance de Faraday pour les hautes températures et déterminé la susceptibilité initiale à partir de mesures isothermes $M_{\mathrm{T}}(H)$. 
A $300 \mathrm{~K}, \chi=2,5 \times 10^{-4} \mathrm{uem} / \mathrm{g}$, soit une valeur nettement supérieure à celle obtenue $\left(0,9 \times 10^{-4} \mathrm{uem} / \mathrm{g}\right)$ par $[6,7]$ dans le cas de l'alliage cristallisé GdAg.

Quand $T$ est supérieur à $160 \mathrm{~K}$, l'alliage suit la loi de Curie Weiss (Fig. 1). Le moment efficace est de $8,36 \mu_{\beta} /$ at., valeur légèrement supérieure à celle attendue dans le cas de l'ion libre $\left(7,95 \mu_{\beta}\right)$ et pratiquement égale à celle trouvée pour l'alliage cristallisé $[6,7]$. La température asymptotique de Curie $\theta_{\mathrm{p}}=+130 \mathrm{~K}$. Ces résultats montrent que les interactions sont ferromagnétiques.

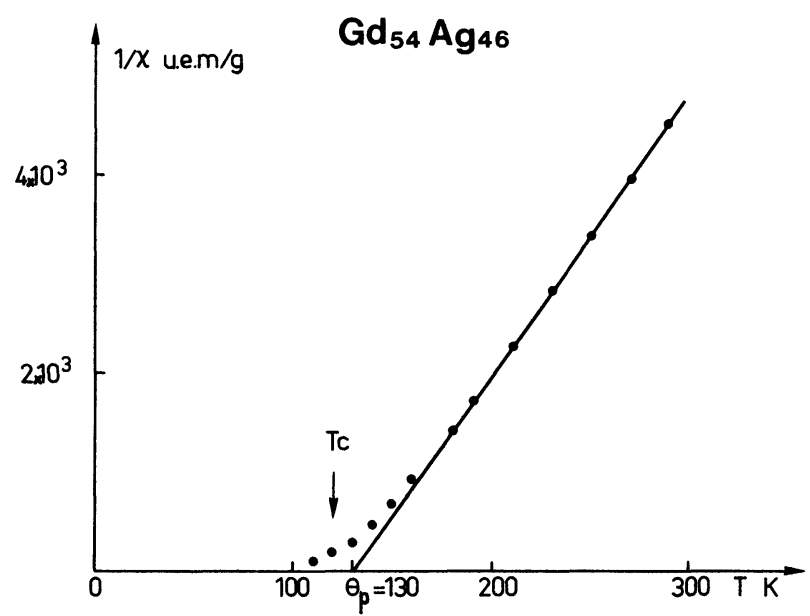

Fig. 1. - Variation de l'inverse de la susceptibilité en fonction de la température.

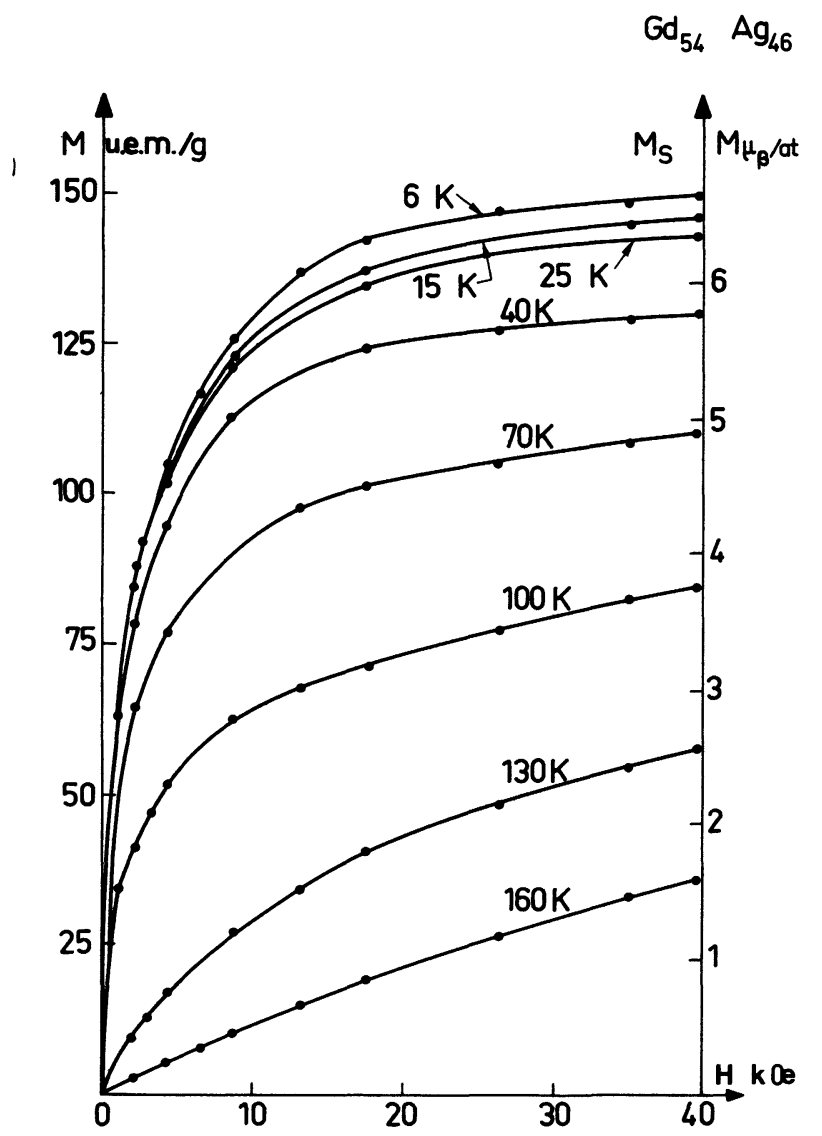

FIG. 2. - Variation isotherme de l'aimantation en fonction du champ appliqué.
Les résultats des mesures isothermes d'aimantation dans des champs allant de 0 à $40 \mathrm{kOe}$ sont représentés sur la figure 2 . Les variations de $M_{\mathrm{T}}(H)$ sont réversibles. A basse température on n'observe pas de rémanence (à quelques uem/g près), ni de champ coercitif (à quelques dizaines de gauss près). A $6 \mathrm{~K}$ et $40 \mathrm{kOe}$, l'aimantation a une valeur $\left(6,7 \mu_{\beta} /\right.$ at. $)$ assez proche de celle attendue $\left(7 \mu_{\beta}\right)$ pour la saturation, mais cette saturation n'est pas encore atteinte, malgré la valeur très forte du champ appliqué à un alliage ferromagnétique. En l'absence d'anisotropie, il est possible d'appliquer la méthode de Landau et de tracer le diagramme d'Arrot $M_{\mathrm{T}}^{2}\left(H_{\text {eff }} / M_{\mathrm{T}}\right)$ (Fig. 3), pour déterminer la température d'ordre. La température de Curie ainsi obtenue est de $122 \mathrm{~K}$. Cette valeur est en très bon accord avec celle obtenue à partir de la courbe donnée par [8]. L'ensemble de ces résultats expérimentaux sont analogues à ceux obtenus avec TbAg amorphe [1] quand $T \geqslant 40 \mathrm{~K}$, c'est-à-dire quand l'anisotropie locale est négligeable devant l'échange.

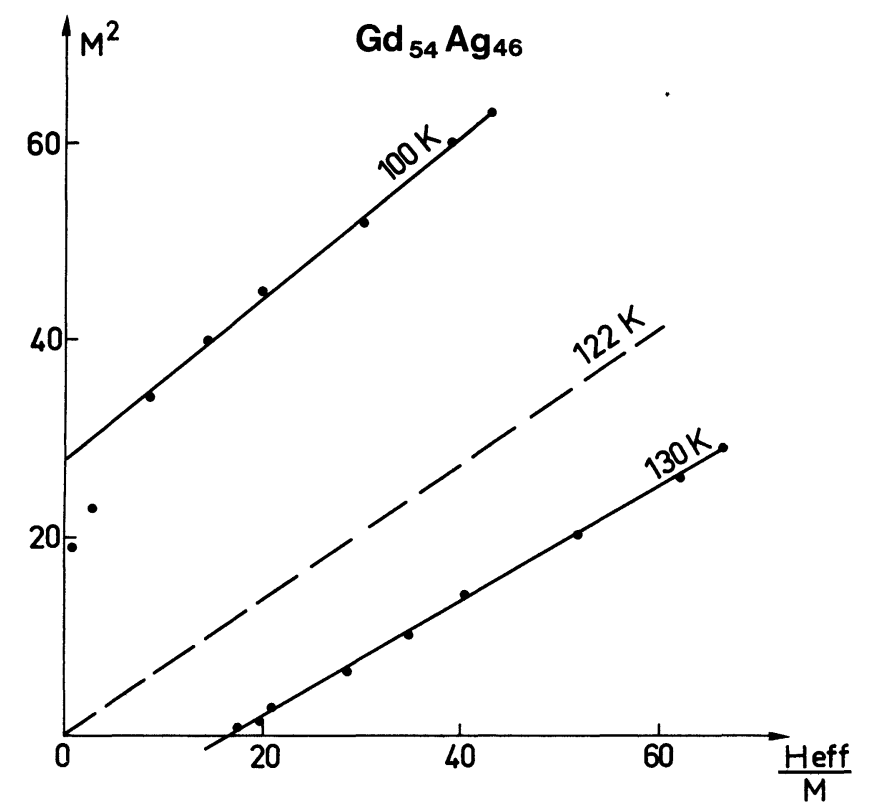

Fig. 3. - Diagramme d'Arrott.

3. Conclusions. - Cette étude montre :

- que l'alliage $\mathrm{GdAg}$ amorphe est ferromagnétique au-dessous de $122 \mathrm{~K}$, tandis que l'alliage cristallisé de même composition est antiferromagnétique. Ce fait a déjà été observé dans le cas TbAg [1];

- que les variations isothermes de l'aimantation en fonction d'un champ magnétique sont réversibles. Rien ne permet de mettre en évidence des phénomènes liés à l'existence de domaines (déplacement de parois, renversement d'aimantation de domaine...). Des mesures en champ faible seront faites pour confirmer ce résultat ;

- qu'un champ appliqué intense est nécessaire pour aligner tous les moments. Ceci implique que 
parmi les interactions d'échange en grande majorité ferromagnétiques, certaines soient antiferromagnétiques. Cette dispersion des interactions est à relier au désordre topologique; les distances interatomiques variant d'un ion à un autre, les interactions magnétiques peuvent varier non seulement en grandeur mais en signe.

La difficulté à atteindre la saturation à basse tem- pérature dans le cas de $\mathrm{TbAg}$, n'est donc pas seulement due à l'importance de l'anisotropie, puisqu'elle est observée avec des alliages où il n'y a pas d'anisotropie. La présence dans un même alliage, à une même température, d'interactions ferromagnétiques et d'interactions antiferromagnétiques entre moments magnétiques portés par des ions de même espèce chimique en est responsable pour une partie.

\section{Bibliographie}

[1] Boucher, B., soumis à Phys. Stat. Sol.

[2] Clark, A. E., Appl. Phys. Lett. 23 (1973) 642.

[3] Rhyne, J. J., Schelleng, J. H. and Koon, N. C., Phys. Rev. B 10 (1974) 4672.

[4] Harris, R., Plische, M. and Zuckermann, M. J., Phys. Rev. Lett. 31 (1973) 160.

[5] Boucher, B., Luzet, D., Sella, C., Compte rendu du $2^{e}$ coll.
Inter. de pulvérisation cathodique, Nice 18/21 mai 1976 (à paraître)

[6] Walline, R. E., Wallace, W. E., J. Chem. Phys. 41 (1964) 3285.

[7] Pierre, J., Pauthenet, R., C. R. Hebd. Séan. Acad. Sci. 260 (1965) 2739.

[8] Hauser, J. J., Phys. Rev. B 12 (1975) 5160. 